\title{
Delimiting the boundaries of sesamoid identities under the network theory framework
}

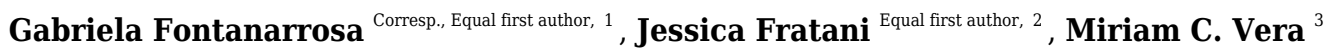 \\ 1 Instituto de Biodiversidad Neotropical (IBN), CONICET-UNT, Yerba Buena, Tucumán, Argentina \\ 2 Unidad Ejecutora Lillo (UEL), CONICET-Fundación Miguel Lillo, San Miguel, Tucumán, Argentina \\ 3 Laboratorio de Genética Evolutiva, Instituto de Biología Subtropical (IBS), CONICET-UNaM, Posadas, Misiones, Argentina \\ Corresponding Author: Gabriela Fontanarrosa \\ Email address: gab.fontanarrosa@gmail.com
}

Sesamoid identity has long been the focus of debate, and how they are linked to other elements of the skeleton has often been considered relevant to its definition. A driving hypothesis of our work was that sesamoids' nature relies deeply on their connections, and thus we propose an explicit network framework to investigate this subject in Leptodactylus latinasus (Anura: Leptodactylidae). Through the dissection of L. latinasus' skeleton, we modeled its anatomical network where skeletal elements were considered nodes while joints, muscles, tendons, and aponeurosis were considered links. The skeletal elements were categorized into canonical skeletal pieces, embedded sesamoids, and glide sesamoids. We inquired about the general network characterization and we have explored further into sesamoid connectivity behavior. We found that the network is structured in a modular hierarchical organization, with five modules on the first level and two modules on the second one. The modules reflect a functional, rather than a topological proximity clustering of the skeleton. The 25 sesamoid pieces are members of four of the first-level modules. Node parameters (centrality indicators) showed that: i) sesamoids are, in general terms, peripheral elements of the skeleton, loosely connected to the canonical bone structures; ii) embedded sesamoids are not significantly distinguishable from canonical skeletal elements; and iii) glide sesamoids exhibit the lowest centrality values and strongly differ from both canonical skeletal elements and embedded sesamoids. The loose connectivity pattern of sesamoids, especially glides, could be related to their evolvability, which in turn seems to be reflected in their morphological variation and facultative expression. Based on the connectivity differences among skeletal categories found in our study, an open question remains: can embedded and glide sesamoids be defined under the same criteria? This study presents a new approach to the study of sesamoid identity and to the knowledge of their morphological evolution. 
Delimiting the boundaries of sesamoid identities under the network theory framework. Gabriela Fontanarrosa ${ }^{1}$ Equal first author, Jessica Fratani ${ }^{2}$ Equal first author, Miriam C. Vera ${ }^{3}$

${ }^{1}$ Instituto de Biodiversidad Neotropical (IBN), UNT-CONICET, Yerba Buena, Tucumán, Argentina.

${ }^{2}$ Unidad Ejecutora Lillo (UEL), CONICET- Fundación Miguel Lillo, San Miguel de Tucumán, Tucumán, Argentina.

${ }^{3}$ Laboratorio de Genética Evolutiva, Instituto de Biología Subtropical (IBS) CONICET- UNaM, Posadas, Misiones, Argentina.

Corresponding Author:

Gabriela Fontanarrosa ${ }^{1}$

Residencia Universitaria Horco Molle (Casa 16), Yerba Buena, Tucumán, 4107, Argentina. Email address: gab.fontanarrosa@gmail.com

\section{Abstract}

Sesamoid identity has long been the focus of debate, and how they are linked to other elements of the skeleton has often been considered relevant to its definition. A driving hypothesis of our work was that sesamoids' nature relies deeply on their connections, and thus we propose an explicit network framework to investigate this subject in Leptodactylus latinasus (Anura: Leptodactylidae). Through the dissection of L. latinasus' skeleton, we modeled its anatomical network where skeletal elements were considered nodes while joints, muscles, tendons, and aponeurosis were considered links. The skeletal elements were categorized into canonical skeletal pieces, embedded sesamoids, and glide sesamoids. We inquired about the general network characterization and we have explored further into sesamoid connectivity behavior. We found that the network is structured in a modular hierarchical organization, with five modules on the first level and two modules on the second one. The modules reflect a functional, rather than a topological proximity clustering of the skeleton. The 25 sesamoid pieces are members of four of the first-level modules. Node parameters (centrality indicators) showed that: i) sesamoids are, in general terms, peripheral elements of the skeleton, loosely connected to the canonical bone structures; ii) embedded sesamoids are not significantly distinguishable from canonical skeletal elements; and iii) glide sesamoids exhibit the lowest centrality values and strongly differ from both canonical skeletal elements and embedded sesamoids. The loose connectivity pattern of sesamoids, especially glides, could be related to their evolvability, which in turn seems to be reflected in their morphological variation and facultative expression. Based on the connectivity differences among skeletal categories found in our study, an open question remains: can embedded and glide sesamoids be defined under the same criteria? This study presents a new approach to the study of sesamoid identity and to the knowledge of their morphological evolution. 
42

43

44

45

46

47

48

49

50

51

52

53

54

55

56

57

58

59

60

61

62

63

64

65

66

67

68

69

70

71

72

73

74

75

76

77

78

79

80

81

82

83

84

85

86

87

\section{Introduction}

Sesamoids have been intriguing scientists since the beginning of the last century (Abdala et al., 2019). No clear consensus exists regarding their identity nor their membership to the canonical skeleton. Sesamoids definitions, often, rely not only on their intrinsic properties but also have strong references to their connections to surrounding tissues (Didomenico et al., 2014; Regnault, Hutchinson \& Jones, 2016). The following definitions serve as examples: "Sesamoids are nodules of cartilage or bone formed in tendons or ligaments, especially where a tendon passes over an angulation of the skeleton" (Hall, 2005); "Sesamoids are periarticular skeletal elements, which initially form in juxtaposition to or independently of bones and joints. They are commonly related to tendons and ligaments (...)" (Abdala et al., 2019). Moreover, beyond those definitions, Jerez, Mangione \& Abdala (2010) categorized sesamoids into four types, also based on their specific relationship to surrounding tissues: (i) embedded sesamoid (ES) (surrounded in all their surfaces by tendinous tissue); (ii) inter-osseous sesamoid (loosely attached to the closest ligaments); (iii) glide sesamoid (GS) (associated to tendons, but not surrounded by them and not fixed to them); and (iv) supporting sesamoid (serving as muscle attachment areas to the corresponding bones).

Since these definitions and classifications commonly refer to sesamoid connections, researchers were considering sesamoids, in an implicit but pervasive way, within a network framework. Network analyses have been increasingly used in the context of comparative vertebrate morphology answering questions about modularity and evolvability, among others (EsteveAltava et al., 2013; Rasskin-Gutman \& Esteve-Altava, 2014; Dos Santos et al., 2017; Diogo et al., 2018). Modular organization allows each structure to evolve semi-independently and promotes evolvability, avoiding deleterious pleiotropic effects (Wagner \& Zhan, 2011; EsteveAltava et al., 2015). Pleiotropic constraints limit evolution through complex and highly controlled global interactions of developmental processes, in which any disturbance would have great consequences (Galis, Metz \& van Alphen, 2018). Based on the same idea, Riedl (1978) proposed that some characters are strongly constrained (less evolvable), while others can change more freely (more evolvable). This difference relies on the burden of a character: as a structure evolves, it develops more relationships with other characters, becoming more and more interconnected and losing its freedom to evolve (Riedl, 1978). The burden theory states that the more the connections, the more the pleiotropic constraints. This can be easily interpreted from anatomical network parameters (Rasskin-Gutman \& Esteve-Altava, 2018).

Among vertebrates, the anuran skeleton is especially interesting to be studied within an anatomical network approach because of their singular anatomy, topologically specialized for locomotion (Dos Santos et al., 2017). While the pelvic girdle and hindlimbs take on a significant role of propulsion, the pectoral girdle and forelimbs are mostly related to landing (Emerson, 1979; 1982; Nauwelaerts \& Aerts, 2006; Astley \& Roberts, 2014). The skeletal pieces are affected by the high mechanical load of jumping locomotion, which could affect the genesis and development of sesamoids pieces (Abdala, Vera \& Ponssa, 2017, Abdala et al., 2019). In this group, sesamoids are present mostly in the joints of the limbs and in the sacral vertebrae (Hoyos, 2003; Ponssa, Goldberg \& Abdala, 2010; Abdala et al., 2019). In particular, Leptodactylus latinasus (Leptodactylidae, Anura), our study case, has a total of 25 sesamoids (Ponssa, Goldberg \& Abdala, 2010; Abdala, Vera \& Ponssa, 2017), that were categorized as ESs and GSs following Jerez, Mangione \& Abdala (2010).

Based on the early and persistent intuition of many authors who worked on sesamoids, a driving hypothesis of our work was that sesamoids' nature relies profoundly on their connections. Thus,

PeerJ reviewing PDF | (2020:03:46890:2:0:NEW 1 Jul 2020) 
88

89

90

91

92

93

94

95

96

97

98

99

100

101

102

103

104

105

106

107

108

109

110

111

112

113

114

115

116

117

118

119

120

121

122

123

124

125

126

127

128

129

130

131

132

133

we propose to study them under an explicit network framework by modeling the L. latinasus skeleton. Based on this model, we explore two main questions related to the topological nature of sesamoids and their impact on the organization of the skeleton. Expressly: 1) How is the network structured?; 2) Do the skeletal pieces categorized as embedded sesamoids, glide sesamoids and canonical skeletal elements differ in network parameters?

\section{Materials \& Methods}

\section{Sample and Data acquisition}

We examined six adult specimens of L. latinasus (one dissected and five cleared and stained). We complemented gross anatomy dissections with several previous anatomic descriptions (Gaupp \& Ecker, 1896; Dunlap, 1960; Nussbaum, 1982; Burton, 1998; Ponssa, Goldberg \& Abdala, 2010; Abdala, Vera \& Ponssa, 2017). Due to the bilateral symmetry of the body, we built an adjacency matrix considering the right half of the body as a proxy of the whole configuration. An adjacency matrix defines the connectivity pattern of the anatomical network by indicating pairs of connected elements. Further details are available in the Supplemental information (SI) (Text S1 and Table S1).

\section{Network construction}

Networks are appropriate mathematical models for tackling the study of biological systems because they are intrinsically collections of entities (nodes) connected through relationships (links). The identification of nodes and links is a critical issue that depends on what we want to know about the modelled system. We modeled the skeletal network of L. latinasus based on the main adjacency matrix. The network model was constructed to inquire on the topological nature of sesamoids within the skeletal system and the anatomical relation among them. Therefore, in our model the nodes represent canonical skeletal pieces and sesamoids (ossified, cartilaginous and fibrocartilaginous). The dorsal fascia was also modeled as a node due to its great extension and role as insertion or origin point for muscles. Since joints, muscles, tendo-muscular units, tendons, and aponeurosis are functionally responsible for the flow of mechanical information among skeletal elements they were considered links in our study. Because all anuran sesamoids are postcranial, the consideration of the whole skull is not informative for our purposes, thus cranial bones were collapsed into a single node. We considered the network as undirected and weighted, considering weights as multiple links.

\section{Network Modularity}

A morphological module is a semi-independent set of densely interconnected skeletal pieces that are only sparsely connected to the rest of the skeleton (Rasskin-Gutman \& Esteve-Altava, 2014; Dos Santos et al., 2017). The number and composition of modules were identified using Order Statistics Local Optimization Method (OSLOM) (Lancichinetti et al., 2011). This community detection algorithm allows the detection of the statistical significance $(b s)$ of modules with respect to random fluctuations. OSLOM takes into account the possibility of homeless nodes (i.e., nodes that are not assigned to any module), as well as the detection of overlapping modules, and the presence of hierarchical organization (Lancichinetti et al., 2011). For our analysis we followed the default options of the OSLOM algorithm, considering bs threshold as 0.1 and allowing the detection of singletons (homeless nodes). Because of the stochastic nature of the OSLOM modularity analysis, results may vary depending on the run (Lancichinetti et al., 2011; Esteve-Altava, 2017). We have reported the most stable modules among multiple runs. 
134

135

136

137

138

139

140

141

142

143

144

145

146

147

148

149

150

151

152

153

154

155

156

157

158

159

160

161

162

163

164

165

166

167

168

169

170

171

172

173

174

175

176

177

178

\section{Sesamoid characterization}

Node parameters

To investigate a possible difference among skeletal categories, we compared node centrality indicators taking into account three skeleton categories $(\mathrm{CSE}=$ canonical skeletal element, $\mathrm{ES}=$ embedded sesamoid, and GS=glide sesamoid). Centrality measures capture the relevance of the position of the individual nodes in the network (Dos Santos et al., 2017). The four most commonly used centrality measures were assessed: 1) Degree: the number of links of a node (Csardi \& Nepusz, 2006); 2) Betweenness: the frequency of events in which a node is located in the shortest path between a pair of nodes (Dos Santos et al., 2017); 3) Closeness: the average length of the shortest path between that particular node and all other nodes in the network (Freeman, 1979); 4) Eigen-centrality: the first eigenvector of the adjacency matrix of the graph (Bonacich, 1987). Nodes with high eigenvector-centrality are those connected to many other nodes, which are, in turn, connected to many others (and so on). Central nodes, under this criterion, belong to centers of big cohesive sets of nodes (Csardi \& Nepusz, 2006). It is important to notice that the centrality values of axial elements might be underestimated because our model included one half of the symmetric body. Lastly, we compared the averages of the aforementioned centrality parameters among the skeletal categories. We calculated the significance of the differences by Kruskal-Wallis tests and then we performed a post-hoc Wilcoxon pairwise comparison test. All the data and the code used to perform our analyses are available in SI (Code S1 and Tables S3-S9).

\section{Results}

\section{Network Characterization}

The anatomical network of Leptodactylus latinasus comprises 102 nodes connected by 328 physical connections (Figs. 1 and 2). Regarding centrality parameters, long bones (especially the tibiafibula), the ilium, and the dorsal fascia stand out by showing the highest centrality values (see SI: Table S3). The module detection algorithm applied (OSLOM) revealed five significant and partial overlapping modules plus two singletons in the first hierarchical level (Figs. 1 and 2, Table 1): the pectoral-forelimb module $(\mathrm{M} 1, b s=0.069)$, the axial-scapular module $(\mathrm{M} 2, b s=$ $0.002)$, the axial-pelvic module $(\mathrm{M} 3, b s=0.081)$, the hindlimb module $(\mathrm{M} 4, b s=0.063)$, and the IV-V toes module (M5, $b s=0.013)$. The fourth vertebra and the urostyle overlap between the two axial modules (M2 and M3). The femur and the ischium overlap between the axial-pelvic and the hindlimb module, and the fibulare overlaps between the hindlimb and the IV-V toes module. The fascia dorsalis and the third phalanx of digit $\mathrm{V}$ of the foot were not assigned to any module in the first hierarchical level (singletons). The configuration of the second hierarchical level presented two modules with a broad overlap of the axial nodes. The third phalanx of digit $\mathrm{V}$ of the foot was also not included in any module in the second hierarchical level.

\section{Sesamoid Patterns}

Sesamoids are widely distributed through the network, being present in all modules except in the axial-pectoral (M2). GSs are arranged peripherally in the network topology. The pectoralforelimb module (M1) includes two ESs and six GSs. In the axial-pelvic module (M3), there are three ESs. The hindlimb module (M4) shows the highest number of sesamoids (nine), and the IV-V toes module contains only GSs (five).

PeerJ reviewing PDF | (2020:03:46890:2:0:NEW 1 Jul 2020) 
179 There are significant differences among node skeletal categories for the all centrality indicators

180 measured. GSs exhibit the lower average values for every centrality indicator, while CSEs

181 exhibit the higher centrality indicator average values except for eigen-centrality. ESs do not

182 differ significantly from CSEs in any of the centrality indicators. GSs are significantly lower

183 both from SEs and from CSEs in degree and closeness. GSs do not differ significantly from ESs

184 regarding betweenness centrality, but they do differ from CSEs in this parameter. GSs do not

185 differ significantly from CSEs in eigen-centrality, but they do differ from ESs in this parameter

186 (Fig. 3; Table 2; see also SI: Table S2 and Figure S1).

\section{Discussion}

Here we present a new approach to the study of sesamoids. Our results agree with the implicit assumptions of many previously proposed sesamoid definitions (see introduction). The connectivity patterns of the skeletal system reflect key properties of sesamoid identity, specially GSs, and suggest that they could have undergone a differential evolutionary mode.

\section{General Network properties}

In the first hierarchical level, and because the skull has been simplified to a single node, the modules are mainly related to the girdles and limbs. Anatomically, the pectoral girdle is divided in a ventral region, composed by the coracoid elements, and in a dorsal region, composed by the scapular elements (Baleeva, 2009). This regionalization could explain why the pectoral girdle is arranged in two modules: the axial-scapular module and the pectoral-forelimb module. In the axial-scapular module, the dorsal elements of the pectoral girdle are more connected to the cranium and to the first vertebrae than to the rest of the pectoral girdle. Indeed, both scapular elements and the cranium are connected by several muscles, such as the M. depressor maxillae which inserts in the lower jaw (Ecker, 1889; Manzano, Moro \& Abdala, 2003). The cartilaginous connection between the scapular and coracoid elements provides a considerable strength of connection while admitting some mobility and decreasing compression forces (Baleeva, 2001). The pectoral-forelimb module is formed by the coracoid elements and the forelimb. This region has an important and complex function absorbing the stress of the impact in the landing phase of the jump (Emerson, 1982). Also, both modules are involved in the complex mechanism of anuran acoustic perception (Lombard \& Straughank, 1974; Kardong, 2012).

The axial-pelvic module is where most of the overlapping occurs in both hierarchical levels of our modularity analysis. This particular configuration of the anuran pelvic girdle seems to represent a topological transitional region between an anterior and posterior region of the network. The elements of this module are deeply affected by developmental changes during metamorphosis from a swimming tadpole into a tailless jumping adult (Soliz \& Ponssa, 2016). These transformations result in a compact and highly integrated vertebral column plus pelvic girdle, epitomized in the network by the membership of the 4th vertebra and the urostyle to both the axial-pectoral and axial-pelvic modules. The femur and the ischium are also simultaneously members of two modules, the axial-pelvic and the hindlimb module. Previous studies have shown that, at the beginning of embryonic formation, the femur develops in close contact with the future acetabulum of the pelvic girdle (Pomikal et al., 2011). Furthermore, functional codependencies exist in the pelvic-hindlimb boundary, in which sequential movement of the hip and leg joints were identified during a typical frog jump (Astley \& Roberts, 2014; Nauwelaerts, Stamhuis \& Aerts, 2005). 
225 The hindlimb module is constituted by pieces of the stylopodium, zeugopodium, and

226 autopodium, most of them are long bones with high centrality values. Long bones have been

227

228 recurrently recovered as central nodes in network analysis of tetrapod limbs (Diogo et al., 2015; Dos Santos et al., 2017). The zeugopodization hypothesis in anurans postulates the elongation of the tibiale and fibulare and a consequent distal shift in the zeugo-autopodial border (Diogo \& Ziermann, 2014; Dos Santos et al., 2017). This could explain why the tibiale and fibulare show high centrality values, that in turn, could be associated with their functional importance as an extra site for muscle attachment (Handrigan \& Wassersug, 2007). Moreover, the fibulare is also part of the IV-V toes module, which is composed of those elements of the foot aligned with this bone. The elements of IV-V toes module also share a common origin of their flexor tendons, which arise from the flexor digitorum brevis superficialis. Contrary to those of the toes I-III, which originate from the aponeurosis plantaris (Gaupp \& Ecker, 1896).

237

238

239

240

241

242

243

244

245

246

247

248

249

250

251

252

253

254

255

256

257

258

259

260

261

262

263

264

265

266

267

268

269

270

\section{Sesamoid Nature, as revealed by node parameters.}

Sesamoids were often conceptually placed outside or beyond the skeleton (Vickaryous \& Olson, 2007). Diogo et al. (2015), indeed modeled many sesamoids as isolated nodes (i.e., without any connection to other pieces) in their strictly skeletal network. On the contrary, our anatomical network does include sesamoids as inner pieces of the skeleton, linked by tendons and muscles to the other skeletal elements. Surprisingly, all of them are unambiguously integrated within four of the modules of the first hierarchical level of the network, being absent only in the axial-pectoral module. Connectivity patterns arrange GSs as peripheral elements of the system, while the ESs are more variably distributed through the network. Both are, in general terms, weakly connected with the canonical pieces of the skeleton as recovered by the centrality indicators. In this sense, sesamoids seem to be lowly burdened structures, sensu Riedl (1978). Connections between elements are established during embryological development (Rasskin-Gutman \& Esteve-Altava, 2018). Sesamoids develop independently and relatively late in comparison to other skeletal elements, only later becoming associated with the primary skeleton (Hall, 2005; Vera, Ponssa \& Abdala, 2015). Although sesamoids may have an imperfect fossil record, probably due to their loose connectivity pattern (this work), their earliest fossil reports predate the Jurassic period (200+ mya) (Vickaryous \& Olson, 2007). By then, most of the skeletal pieces would have been evolving for at least 420 million years (Ravi \& Venkatesh, 2008). In turn, sesamoids' high diversity in size, shape, number, and distribution (Abdala et al., 2019) could be a consequence of being low burdened structures. In fact, high rates of evolution of sesamoid bones were reported by Baum \& Smith (2013). Thus, the loose connectivity pattern characterizing sesamoids seems to be a consequence of their delay in ontogeny and phylogeny.

The facultative expression of many sesamoids in the phenotype as a response to continuous mechanical stress (i.e., epigenetic influence) (Abdala \& Ponssa, 2012; Abdala et al., 2019) could be, at least in part, a consequence of the low burden of these skeletal pieces. Perturbations on sesamoids development is unlikely to be accompanied by systemic consequences for an organism, as could be the case of perturbations on the development of canonical elements. As lowly constrained pieces, sesamoids may be labile evolutionary elements, with a relatively high capacity of generating heritable phenotypic variation (Kirschner \& Gerhart, 1998), in turn the path of the appearance of evolutionary novelties would be facilitated. This rationale is in accordance with the dynamic model stated by Abdala et al. (2019) in which sesamoids are proposed as a source of new skeletal morphologies available to natural selection processes.

Peerj reviewing PDF | (2020:03:46890:2:0:NEW 1 Jul 2020) 
271

272

273

274

275

276

277

278

279

280

281

282

283

284

285

286

287

288

289

290

291

292

293

294

295

296

297

298

299

300

301

302

303

304

305

306

307

308

309

310

311

312

313

314

315

316

\section{II-1. Embedded sesamoids.}

ESs centralities values were not significantly different from CSEs elements, but they resulted to be significantly more central than those of the GSs (except for betweenness). The fact of being embedded in the connective tissue of the most powerful muscles of the limbs (Jerez, Mangione \& Abdala, 2010), which were considered as network links, straightforwardly contributes to the higher centrality values of ESs when compared to GSs. ESs are distributed in three modules related to the limbs and the pelvic girdle (M1, M3, and M4), and absent from axial-scapular and IV-V toes modules (M2 and M5). Most ESs are included within the hindlimb module, coincidentally, this module is subject to the highest mechanical forces during the take-off phase of the jump (Nauwelaerts \& Aerts, 2006). The palmar sesamoid (pectoral-forelimb module) showed a notably high betweenness value among embedded sesamoids, and surprisingly similar to top-ranked canonical elements (SI: Table S3). This could be associated with the fact that the palmar sesamoid is embedded in the m. flexor digitorum longus which is the source of the flexor tendons of digits II-V (Ponssa, Goldberg \& Abdala 2010; Diogo \& Abdala, 2010).

It is logical to think that nearby pieces will tend to be more connected than distant pieces. Thus, a correspondence between network modules and euclidean regions of the body is expected (Dos Santos et al., 2017). Sesamoids, in general, have links other than joints connecting them to other skeletal pieces (Vickaryous \& Olson, 2007; Table S1). This property allows them to defy the general proximity imposition, in such a way that they are able to share a module with spatially distant pieces. In fact, the patella and the graciella sesamoids, located in the knee joint, are coopted by a more proximal module (axial-pelvic module) instead of the hindlimb module, as we could expect following a spatial neighborhood criterion. These sesamoids constitute the only elements in the network with such kind of behavior. This pattern could be explained by their remote connection with the pelvic girdle by the cruralis and the gracilis major muscles, respectively, which form a set of muscles required for the extension and flexion of the knee joint (Abdala, Vera \& Ponssa, 2017; Table S1). Additionally, Eyal et al. (2015) show that, in mice, the patella arises as part of the femur but from a distinct pool of progenitors. Thus, probably, the patella membership to the axial-pelvic module can be explained by complex cellular and genetic mechanisms during the morphogenesis process.

\section{II-2. Glide sesamoids}

Centrality indicators mainly segregated the GSs from the other skeletal categories. Frequently, GSs are implicitly excluded from sesamoid conceptual delimitation, due to definitions typically consider sesamoids as elements surrounded by tendinous or ligamentous structures (e.g., Hall, 2005; “(...) sesamoids are independent ossifications/chondrifications within tendons"). Moreover, developmental evidence has shown that although ESs and GSs share the same progenitor cells, they have different developmental signaling paths (Eyal et al., 2019). Glide sesamoids are significatively less connected than ESs and CSEs when comparing degree and closeness. A different trend is revealed by the eigen-centrality indicator, which is similar between ESs and CSEs, but distinguishes the two sesamoid categories highlighting the particularities of GSs. Low eigen-centrality indicates that not only GSs, but also that their neighbor nodes have few connections. The unusually low centrality indicators of GSs could be a proxy of a high evolvability of those bones following the burden theory (Rasskin-Gutman \& Esteve-Altava, 2018). Indeed, high intraspecific variation in number and morphology has been reported in glides (Ponssa, Goldberg \& Abdala, 2010). Therefore, low connectivity could represent an alternative strategy to modularity in order to increase evolvability. 
317 The anatomical distribution, shape, constitution, and the paired condition of GSs of the forelimb

318 in L. latinasus (Ponssa, Goldberg \& Abdala, 2010), is similar to those of paraphalangeal

319 elements that characterize many pad-bearing geckos (Squamata). The multiple origins of

320

321

322

323

324

325

326

327

328

329

330

331

332

333

334

335

336

337

338

339

340

341

342

343

344

345

346

347

348

349

350

351

352

353

354

355

356

357

358

359

360

361 paraphalanges plus their extremely variable morphology (Wellborn, 1933; Russell \& Bauer, 1988; Gamble et al., 2012, Fontanarrosa, Daza \& Abdala, 2018) supports the idea of their lability in evolutionary terms. Curiously, lizards that lack paraphalanges also lack GSs related to interphalangeal joints (Fontanarrosa, 2018). Additional network analysis, modeling a species with paraphalanges, would most likely indicate that they are relatively disconnected structures of the main skeleton. Connectivity patterns have long been a criterion for the recognition of homologies (Geoffroy Saint-Hilaire, 1818). Thus, identifying common connections to specific elements, could reveal putative homologous structures through distant related lineages such as paraphalanges and GSs. Furthermore, dissimilar connectivity patterns between ESs and GSs found in this study suggest that they may not be members of the same hierarchical category. Future studies based on complementary sources of evidence, such as development or evolution, are required to test this hypothesis.

\section{Conclusions}

Here we presented a new approach to the study of sesamoid identity and hope to contribute to the current research on their morphological evolution. Our findings raise interesting questions to be investigated in other species of tetrapods, as well as by complementary areas of research, such as developmental or evolutionary biology. Multiple sesamoid definitions based on their relations with canonical bones and connective tissue were calling for their explicit framing under network theory. After performing an anatomical network analysis of a model anuran species $(L$. latinasus), we inquired first on the general topology, and more specifically on sesamoid connectivity patterns. The main conclusions that emerged from this approach are:

1. The skeletal elements were clustered in five modules that reflect a functional organization. Four modules contain at least one sesamoid.

2. Sesamoids, in general terms, are peripheral elements of the network, with few connections to the canonical skeleton. This could explain their considerable variation on size, shape, number, distribution and high evolvability. These results support the hypothesis of sesamoids as morphological innovations generators.

3. Embedded sesamoids have, on average, similar centrality values to the canonical skeletal elements. These sesamoids are surrounded by connective tissue, thus are prone to have more connections than glide sesamoids. While glides are adjacent to tendons, but not fixed to them.

4. Glide sesamoids have the lowest values for every centrality indicator measured, when compared to the other skeletal categories.

5. Similarities between embedded sesamoids and canonical bones, in addition to glides' own singularities, leave an open question as to whether all embedded and glide sesamoids have the same nature.

\section{Acknowledgements}

Conversations and exchanges of ideas with Virginia Abdala (IBN, CONICET, Argentina) helped us considerably. We also thank Borja Esteve-Altava, Mario Hoyos, and an anonymous reviewer for their suggestions to improve the manuscript. We thank Dr. Diego Baldo, curator of the herpetological collection of the Laboratorio de Genética Evolutiva of Instituto de Biología

362 Subtropical, for providing the study material. 
Abdala V, Ponssa ML. 2012. Life in the slow lane: The effect of reduced mobility on tadpole limb development. Anatomical Records 295(1):5-17. Abdala V, Vera MC, Ponssa ML. 2017. On the Presence of the Patella in Frogs. Anatomical Records 300:1747-1755. Abdala V, Vera MC, Amador LI, Fontanarrosa G, Fratani J, Ponssa L. 2019. Sesamoids in tetrapods: the origin of new skeletal morphologies. Biological Reviews 94(6):2011-2032. Astley HC, Roberts TJ. 2014. The mechanics of elastic loading and recoil in anuran jumping. Journal of Experimental Biology 217(24):4372-4378. Baum DA, Smith SD. 2013. Using trees to study character evolution. In: Baum DA, Smith SD, eds. Tree Thinking: An Introduction to Phylogenetic Biology. Colorado: Roberts and Company Publishers, 305-348. Bonacich P. 1987. Power and Centrality: A Family of Measures. American Journal of Sociology 92:1170-1182.

Baleeva NV. 2001. Formation of the scapular part of the pectoral girdle in anuran larvae. Russian Journal of Herpetology 8(3):195-204. Baleeva NV. 2009. Formation of the coracoid region of the anuran pectoral girdle. Russian Journal of Herpetology 16(1):41-50.

Burton TC. 1998. Variation in the hand and superficial throat musculature of Neotropical leptodactylid frogs. Herpetologica 53-72.

Csardi G, Nepusz T. 2006. The igraph software package for complex network research. InterJournal, complex systems 1695(5):1-9. of Sesamoid Apparatus during the Lapidus Procedure: A Novel Approach. The Journal of Foot \& Ankle Surgery 53:248-251.

Diogo R, Abdala V. 2010. Pectoral and forelimb muscles of limbed amphibians and reptiles. In:

392

393

Diogo R, Abdala V, eds. Muscle of Vertebrates - Comparative Anatomy, Evolution, Homologies and Development. Florida: CRC Press, 359-420.

Diogo R, Ziermann JM. 2014. Development of fore- and hindlimb muscles in frogs,

394

395 morphogenesis, homeotic transformations, digit reduction, and the forelimb-hindlimb enigma. Journal of Experimental Zoology 322:86-105.

Diogo R, Esteve-Altava B, Smith C, Boughner JC, Rasskin-Gutman D. 2015. Anatomical network comparison of human upper and lower, newborn and adult, and normal and abnormal limbs, with notes on development, pathology and limb serial homology vs. homoplasy. PLoS One 10(10): e0140030 DO: 10.1371/journal.pone.0140030.

Diogo R, Molnar JL, Rolian C, Esteve-altava B. 2018. First anatomical network analysis of foreand hindlimb musculoskeletal modularity in bonobos, common chimpanzees, and humans. Scientific reports 8(1):1-9 DOI:10.1038/s41598-018-25262-6. Dos Santos D, Fratani J, Ponssa ML, Abdala V. 2017. Network architecture associated with the highly specialized hindlimb of frogs. PLoS One 12:1-17.

Dunlap DG. 1960. The comparative myology of the pelvic appendage in the Salientia. Journal of Morphology 106(1):1-76.

Ecker, A. 1889. The anatomy of the frog (Vol. 2). Clarendon Press. 
408 Emerson SB. 1979. The 1lio-sacral articulation in frogs: form and function. Biological Journal of 409 the Linnean Society 11:153-168.

410 Emerson SB. 1982. Frog postcranial morphology: identification of a functional complex. Copeia 411 1982:603-613.

412 Esteve-Altava B, Marugán-Lobón J, Botella H, Bastir M, Rasskin-Gutman D. 2013. Grist for

413 Riedl's mill: A network model perspective on the integration and modularity of the human skull.

414 Journal of Experimental Zoology Part B: Molecular and Developmental Evolution 320:489-500.

415 Esteve-Altava B, Diogo R, Smith C, Boughner JC, Rasskin-Gutman D. 2015. Anatomical

416 networks reveal the musculoskeletal modularity of the human head. Scientifics Reports 5:1-6.

417 Esteve-Altava B. 2017. Challenges in identifying and interpreting organizational modules in

418 morphology. Journal of Morphology, 278: 960-974.

419 Eyal S, Blitz E, Shwartz Y, Akiyama H, Ronen S, Zelzer E. 2015. On the development of the 420 patella. Development 142:1-9 DOI: 10.1242/dev.121970.

421 Eyal S, Rubin S, Krief S, Levin L, Zelzer E. 2019. Common cellular origin and diverging

422 developmental programs for different sesamoid bones. Development 146:dev167452

423 DOI:10.1242/dev.167452.

424 Fontanarrosa G. 2018. La Evolución de la Mano de Squamata: Perspectivas Ecológicas y

425 Funcionales. D. Phil. Thesis, Universidad Nacional de Tucumán.

426 Fontanarrosa G, Daza JD, Abdala V. 2018. Cretaceous fossil gecko hand reveals a strikingly

427 modern scansorial morphology: qualitative and biometric analysis of an amber-preserved lizard

428 hand. Cretaceous Research 84:120-133.

429 Freeman LC. 1979. Centrality in Social Networks I: Conceptual Clarification. Social Networks

$430 \quad 1: 215-239$.

431 Galis F, Metz JA, van Alphen JJ. 2018. Development and evolutionary constraints in animals.

432 Annual Review of Ecology, Evolution, and Systematics 49:499-522.

433 Gamble T, Greenbaum E, Jackman TR, Russell AP, Bauer AM. 2012. Repeated origin and loss

434 of adhesive toepads in Geckos. PLoS One 7(6): e39429 DOI: 10.1371/journal.pone.0039429.

435 Gaupp E, Ecker A. 1896. A. Ecker's und R. Wiedersheim's Anatomie des Frosches. 1. Lehre vom

436 Skelet und vom Muskelsystem. Vieweg.

437 Geoffroy Saint-Hilaire, E. 1818. Philosophie anatomique. J. B. Baillie`re, Paris.

438 Hall BK. 2005. Tendons and Sesamoids. In: Hall BK, ed. Bones and Cartilages - Developmental

439 and Evolutionary Skeletal Biology. Cambridge: Academic Press, 115-123 DOI:10.1016/B978-

440 012319060-4/50011-7.

441 Handrigan GR, Wassersug RJ. 2007. The anuran Bauplan: a review of the adaptive,

442 developmental, and genetic underpinnings of frog and tadpole morphology. Biological Reviews

443 82:1-25.

444 Hoyos JM. 2003. Additions to our knowledge of anuran sesamoids. Herpetological Review

$445 \quad 34: 112-116$.

446 Jerez A, Mangione S, Abdala V. 2010. Occurrence and distribution of sesamoid bones in

447 squamates: a comparative approach. Acta Zoologica Stockholm 91:295-305.

448 Kardong, K. V. (2012). Vertebrates: comparative, function, evolution. Washington State Univ, $44933,81-87$.

450 Kirschner M, Gerhart J. 1998. Evolvability. Proceedings of the National Academy of Sciences 451 95(15):8420-8427.

452 Lancichinetti A, Radicchi F, Ramasco JJ, Fortunato S. 2011. Finding statistically significant

453 communities in networks. PloS one 6(4):e18961. doi:10.1371/journal.pone.0018961. 
454 Lombard RE, Straughan IR. 1974. Functional aspects of anuran middle ear structures. Journal of 455 Experimental Biology 61(1):71-93.

456 Manzano AS, Moro S, Abdala V. 2003. The depressor mandibulae muscle in Anura. Alytes. 20

457 (3-4) 93-131.

458 Nauwelaerts S, Stamhuis E, Aerts P. 2005. Swimming and jumping in a semi-aquatic frog.

459 Animal Biology 55(1): 3-15.

460 Nauwelaerts S, Aerts P. 2006. Take-off and landing forces in jumping frogs. Journal of

461 Experimental Biology 209:66-77.

462 Nussbaum RA. 1982. Heterotopic Bones in the Hindlimbs of Frogs of the Families Pipidae,

463 Ranidae and Sooglossidae. Herpetologica 38:312-320.

464 Pomikal C, Blumer R, Streicher J. 2011. Four-dimensional analysis of early pelvic girdle

465 development in Rana temporaria. Journal of morphology, 272(3): 287-301.

466 Ponssa ML, Goldberg J, Abdala V. 2010 Sesamoids in anurans: New data, old issues.

467 Anatomical Records 293:1646-1668.

468 R Core Team. 2019. R: A language and environment for statistical computing. R Foundation for 469 Statistical Computing, Vienna, Austria. URL https://www.R-project.org/.

470 Rasskin-Gutman D, Esteve-Altava DRB. 2014. Connecting the Dots: Anatomical Network

471 Analysis in Morphological EvoDevo. Biological Theory 9:178-193.

472 Rasskin-Gutman D, Esteve-Altava DRB. 2018. Concept of Burden in Evo-Devo. In: Nuno de la

473 Rosa L, Müller G, eds. Evolutionary Developmental Biology. Berlin: Springer, 1-11. DOI:

474 10.1007/978-3-319-33038-9_48-1.

475 Ravi V, Venkatesh B. 2008. Rapidly evolving fish genomes and teleost diversity. Current

476 Opinion in Genetics \& Development 18(6):544-550.

477 Regnault S, Hutchinson JR, Jones ME. 2016. Sesamoid bones in tuatara (Sphenodon punctatus)

478 investigated with X-ray microtomography, and implications for sesamoid evolution in

479 Lepidosauria. Journal of morphology 278(1):62-72.

480 Riedl R. 1978. Order in living organisms: a systems analysis of evolution. New York: Wiley.

481 Russell AP, Bauer AM. 1988. Paraphalangeal elements of gekkonid lizards - a comparative

482 survey. Journal of Morphology 197:221-240.

483 Soliz M, Ponssa ML. 2016. Development and morphological variation of the axial and

484 appendicular skeleton in Hylidae (Lissamphibia, Anura). Journal of morphology 277(6):786-813.

485 Vera MC, Ponssa ML, Abdala V. 2015. Further Data on Sesamoid Identity from Two Anuran

486 Species. Anatomical Records 298(8):1376-1394.

487 Vickaryous MK, Olson WM. 2007. Sesamoids and ossicles in the appendicular skeleton. In: Hall

488 B, ed. Fins into limbs: evolution, development and transformation. Chicago: University of

489 Chicago Press, 323-341.

490 Wagner GP, Zhang J. 2011. The pleiotropic structure of the genotype-phenotype map: the

491 evolvability of complex organisms. Nature Reviews Genetics 12(3):204-213.

492 Wellborn V. 1933. Vergleichende osteologische Untersuchungen an Geckoniden, Eublephariden 493 und Uroplatiden. Berlin: Sitzung der Gesellschaft Naturfreunde.

494

495

496

497

498 Figure 1. The anatomical network of Leptodactylus latinasus with an inset (i) providing a

499 schematic representation of the network relative to the species body. Links are weighted by the

PeerJ reviewing PDF | (2020:03:46890:2:0:NEW 1 Jul 2020) 
500 number of connections, and nodes are colored according to membership to modules: pectoral501 forelimb module (green), axial-scapular module (orange), axial-pelvic module (yellow),

502 hindlimb module (blue), IV-V toes module (purple), and homeless pieces (grey). Mix coloured

503 nodes are simultaneously members of two modules. Different shapes distinguish among skeletal

504 categories of nodes: non-sesamoids, glide sesamoids, and embedded sesamoids. ID numbers are 505 shown in Table 1.

Figure 2. Leptodactylus latinasus skeleton with the pieces colored according to network modules. A. Dorsal view. B. ventral view. The fascia dorsalis (pectoral-forelimb module) is represented in a non-saturated grey color to allow the visualization of overlapped structures.

510 Color references: pectoral-forelimb module (green), axial-scapular module (orange), axial-pelvic 511 module (yellow), hindlimb module (blue), IV-V toes module (purple), and homeless pieces 512 (grey). Mix colored pieces are simultaneously members of two modules.

513

Figure 3. Schematic representation and node parameter values of each node type in the network. A. Ventral view of the left hand of Leptodactylus latinasus showing the canonical bones (light purple), the palmar sesamoid (light blue) interphalangeal and metacarpophalangeal glide sesamoids (pink). B. Detail of the palmar sesamoid (embedded type) with the flexor tendons of the digits. C. Detail of an interphalangeal glide associated with a flexor tendon. D. Boxplots of degree values by node category. E. Boxplots of betweenness values by node category. F. Boxplots of closeness values by node category. G. Boxplots of eigen-centrality values by node category. Abbreviations: CSE: Canonical Skeletal Element (light purple); ES: embedded sesamoid (light blue); GS: glide sesamoid (pink).

\section{Table Captions}

Table 1. Modules composition. ID node numbers are superscripted and elements highlighted in bold are included in more than one module. Abbreviations: F: finger FP: forelimb phalanx, HP: hindlimb phalanx, (f) forelimb, (h): hindlimb, T: toe, V: vertebra.

Table 2. Sesamoid centrality indicators summary. Abbreviations: Cat.: Sesamoid category following Jerez, Mangione \& Abdala (2010); M: Module; D: Degree; B: Betweenness; C: Closeness; E-C: Eigen centrality. 


\section{Table 1 (on next page)}

Modules composition.

ID node numbers are superscripted and elements highlighted in bold are included in more than one module. Abbreviations: F: finger FP: forelimb phalanx, HP: hindlimb phalanx, (f) forelimb, (h): hindlimb, T: toe, V: vertebra. 
Table 1. Modules composition. ID node numbers are superscripted and elements highlighted in bold are included in more than one module. F: finger FP: forelimb phalanx, HP: hindlimb phalanx, (f) forelimb, (h): hindlimb, T: toe, V: vertebra.

\begin{tabular}{|c|c|c|c|}
\hline Module & Canonical skeletal elements & Embedded Sesamoid & Glide Sesamoid \\
\hline $\begin{array}{l}\text { Pectoral-forelimb } \\
\text { (M1 - green) }\end{array}$ & 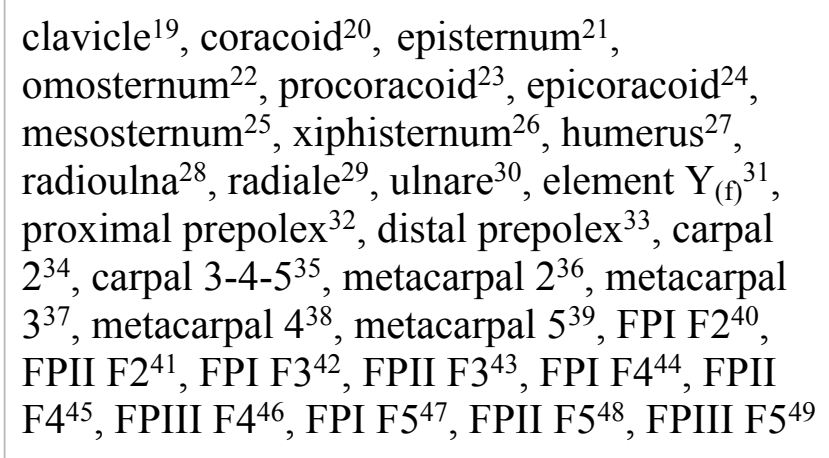 & $\begin{array}{l}\text { palmar sesamoid }^{95} \\
\text { pararadial }^{96}\end{array}$ & $\begin{array}{l}\text { metacarpal glide F297, } \\
\text { metacarpal glide F3 }{ }^{98} \text {, } \\
\text { metacarpal glide F4 }{ }^{99} \text {, } \\
\text { interphalangeal glide FPII-I } \\
\mathrm{F}^{100}{ }^{10} \text { metacarpal glide F5 }{ }^{101} \text {, } \\
\text { interphalangeal glide FPII-I } \\
\text { F5 }{ }^{102}\end{array}$ \\
\hline $\begin{array}{l}\text { Axial-scapular } \\
\text { (M2 - red) }\end{array}$ & 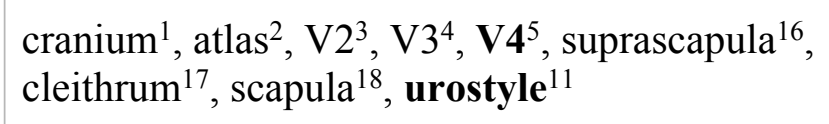 & - & - \\
\hline $\begin{array}{l}\text { Hindlimb module } \\
\text { (M4 - blue) }\end{array}$ & 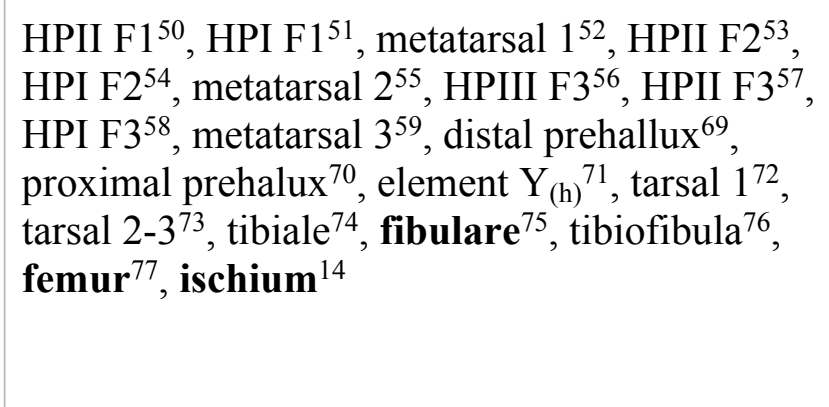 & $\begin{array}{l}\text { cartilago sesamoide }{ }^{87} \text {, plantar } \\
\text { sesamoid I of the plantar } \\
\text { aponeurosis } 88 \text {, plantar } \\
\text { sesamoid II of the plantar } \\
\text { aponeurosis } 89 \text {, OS Sesamoides } \\
\text { tarsale }^{90}, \text { plantar } \\
\text { sesamoid of the flexor } \\
\text { digitorum }^{91}\end{array}$ & $\begin{array}{l}\text { metatarsal glide } F 1^{78} \text {, metatarsal } \\
\text { glide F2 }{ }^{79} \text {, interphalangeal glide } \\
\text { HPII-I F3 }{ }^{80} \text {, metatarsal glide } \\
\text { F3 }\end{array}$ \\
\hline
\end{tabular}




\begin{tabular}{|c|c|c|c|}
\hline $\begin{array}{l}\text { IV-V toes module } \\
\text { (M5 - purple) }\end{array}$ & $\begin{array}{l}\text { HPIV F4 }{ }^{60}, \text { HPIII F4 }{ }^{61}, \text { HPII F4 }{ }^{62}, \text { HPI F4 }{ }^{63} \text {, } \\
\text { metatarsal } 4^{64}, \text { HPII F5 }{ }^{66}, \text { HPI F }^{67} \text {, metatarsal } \\
5^{68}, \text { fibulare }^{75}\end{array}$ & - & $\begin{array}{l}\text { interphalangeal glide HPIII-II } \\
\text { F4 }{ }^{82} \text {, interphalangeal glide } \\
\text { HPII-I F4 }{ }^{83} \text {, metatarsal glide } \\
\text { F4 }{ }^{84} \text {, interphalangeal glide } \\
\text { HPII-I F5 }{ }^{85} \text {, metatarsal glide } \\
\text { F5 }\end{array}$ \\
\hline Singletons & fascia dorsalis $^{92}$, HPIII F565 & - & - \\
\hline
\end{tabular}




\section{Table 2 (on next page)}

Sesamoid centrality indicators summary

Abbreviations: Cat.: Sesamoid category following Jerez, Mangione \& Abdala (2010); M:

Module; D: Degree; B: Betweenness; C: Closeness; E-C: Eigen centrality. 
1 Table 2. Sesamoid centrality indicators summary. Abbreviation: Cat. : Sesamoid category

2 following Jerez, Mangione \& Abdala (2010); M: Module; D: Degree; B: Betweenness; C:

3 Closeness; E-C: Eigen centrality.

4

\begin{tabular}{|c|c|c|c|c|c|c|c|c|}
\hline Sesamoids & ID & Muscle/tendon & Cat. & D & $\mathbf{C}$ & B & $\mathbf{E}$ & $\mathbf{M}$ \\
\hline Cartilago sesamoide & 87 & $\begin{array}{l}\text { plantaris } \\
\text { profundus }\end{array}$ & Embedded & 6 & 0.272 & 4.183 & 0.157 & 4 \\
\hline $\begin{array}{l}\text { Glide interphalangeal } \\
\text { II-I of finger IV }\end{array}$ & 100 & interphalangeal & Glide & 2 & 0.189 & 0.000 & 0.000 & 1 \\
\hline $\begin{array}{l}\text { Glide interphalangeal } \\
\text { II-I of finger V }\end{array}$ & 102 & interphalangeal & Glide & 2 & 0.186 & 0.000 & 0.000 & 1 \\
\hline $\begin{array}{l}\text { Glide interphalangeal } \\
\text { II-I of toe IV }\end{array}$ & 83 & $\begin{array}{l}\text { flexor digitii } \\
\text { brevis }\end{array}$ & Glide & 2 & 0.221 & 0.467 & 0.031 & 5 \\
\hline $\begin{array}{c}\text { Glide interphalangeal } \\
\text { II-I of toe } \mathrm{V}\end{array}$ & 85 & $\begin{array}{l}\text { flexor digitii } \\
\text { brevis }\end{array}$ & Glide & 2 & 0.221 & 0.583 & 0.024 & 5 \\
\hline $\begin{array}{l}\text { Glide interphalangeal } \\
\text { II-Iof toe III }\end{array}$ & 80 & $\begin{array}{l}\text { flexor digitii } \\
\text { brevis }\end{array}$ & Glide & 2 & 0.221 & 0.726 & 0.025 & 4 \\
\hline $\begin{array}{l}\text { Glide interphalangeal } \\
\text { III-II of toe IV }\end{array}$ & 82 & $\begin{array}{l}\text { flexor digitii } \\
\text { brevis }\end{array}$ & Glide & 2 & 0.217 & 0.000 & 0.013 & 5 \\
\hline Glide of metacarpal II & 97 & lumbricalis brevis & Glide & 2 & 0.221 & 0.000 & 0.000 & 1 \\
\hline $\begin{array}{c}\text { Glide of metacarpal } \\
\text { III }\end{array}$ & 98 & lumbricalis brevis & Glide & 2 & 0.221 & 2.042 & 0.000 & 1 \\
\hline $\begin{array}{l}\text { Glide of metacarpal } \\
\text { IV }\end{array}$ & 99 & lumbricalis brevis & Glide & 2 & 0.221 & 1.930 & 0.000 & 1 \\
\hline Glide of metacarpal V & 101 & lumbricalis brevis & Glide & 3 & 0.226 & 4.404 & 0.000 & 1 \\
\hline Glide of metatarsal I & 78 & $\begin{array}{c}\text { flexor digitii } \\
\text { brevis }\end{array}$ & Glide & 2 & 0.223 & 0.000 & 0.051 & 4 \\
\hline Glide of metatarsal II & 79 & $\begin{array}{l}\text { flexor digitii } \\
\text { brevis }\end{array}$ & Glide & 2 & 0.223 & 0.309 & 0.047 & 4 \\
\hline Glide of metatarsal III & 81 & $\begin{array}{l}\text { flexor digitii } \\
\text { brevis }\end{array}$ & Glide & 2 & 0.224 & 0.610 & 0.044 & 4 \\
\hline Glide of metatarsal IV & 84 & $\begin{array}{l}\text { flexor digitii } \\
\text { brevis }\end{array}$ & Glide & 2 & 0.225 & 0.450 & 0.045 & 5 \\
\hline Glide of metatarsal V & 86 & $\begin{array}{l}\text { flexor digitii } \\
\text { brevis }\end{array}$ & Glide & 2 & 0.221 & 1.444 & 0.037 & 5 \\
\hline Graciella & 94 & gracilis major & Embedded & 3 & 0.271 & 76.943 & 0.097 & 3 \\
\hline OS sesamoides tarsale & 90 & Achilles tendon & Embedded & 7 & 0.277 & 4.804 & 0.216 & 4 \\
\hline Palmar sesamoid & 95 & $\begin{array}{l}\text { Flexor digitorum } \\
\text { communis - }\end{array}$ & Embedded & 10 & 0.275 & $\begin{array}{r}535.51 \\
3\end{array}$ & 0.001 & 1 \\
\hline
\end{tabular}




\begin{tabular}{|c|c|c|c|c|c|c|c|c|}
\hline & & Flexor plate & & & & & & \\
\hline Pararadial & 96 & $\begin{array}{l}\text { Extensor carpi } \\
\quad \text { radialis }\end{array}$ & Embedded & 2 & 0.259 & 0.000 & 0.001 & 1 \\
\hline Patella & 93 & knee aponeurosis & Embedded & 3 & 0.278 & 0.000 & 0.160 & 3 \\
\hline $\begin{array}{l}\text { Plantar } \\
\text { sesamoid of the flexor } \\
\text { digitorum }\end{array}$ & 91 & $\begin{array}{l}\text { flexor dig brevis } \\
\text { superficialis }\end{array}$ & Embedded & 6 & 0.273 & 68.815 & 0.167 & 4 \\
\hline $\begin{array}{c}\text { Plantar sesamoid I of } \\
\text { the plantar } \\
\text { aponeurosis }\end{array}$ & 88 & $\begin{array}{c}\text { plantar } \\
\text { aponeurosis }\end{array}$ & Embedded & 11 & 0.281 & 61.837 & 0.297 & 4 \\
\hline $\begin{array}{c}\text { Plantar sesamoid II of } \\
\text { the plantar } \\
\text { aponeurosis }\end{array}$ & 89 & $\begin{array}{c}\text { plantar } \\
\text { aponeurosis }\end{array}$ & Embedded & 11 & 0.281 & 56.528 & 0.308 & 4 \\
\hline Sacral sesamoid & 13 & $\begin{array}{l}\text { internal ligament } \\
\text { of the sacrum }\end{array}$ & Embedded & 2 & 0.277 & 0.000 & 0.027 & 3 \\
\hline
\end{tabular}

5 


\section{Figure 1}

The anatomical network of Leptodactylus latinasus with an inset (i) providing a schematic representation of the network relative to the species body

Links are weighted by the number of connections, and nodes are colored according to membership to modules: pectoral-forelimb module (green), axial-scapular module (orange), axial-pelvic module (yellow), hindlimb module (blue), IV-V toes module (purple), and homeless pieces (grey). Mix coloured nodes are simultaneously members of two modules. Different shapes distinguish among skeletal categories of nodes: non-sesamoids, glide sesamoids, and embedded sesamoids. ID numbers are shown in Table 1.

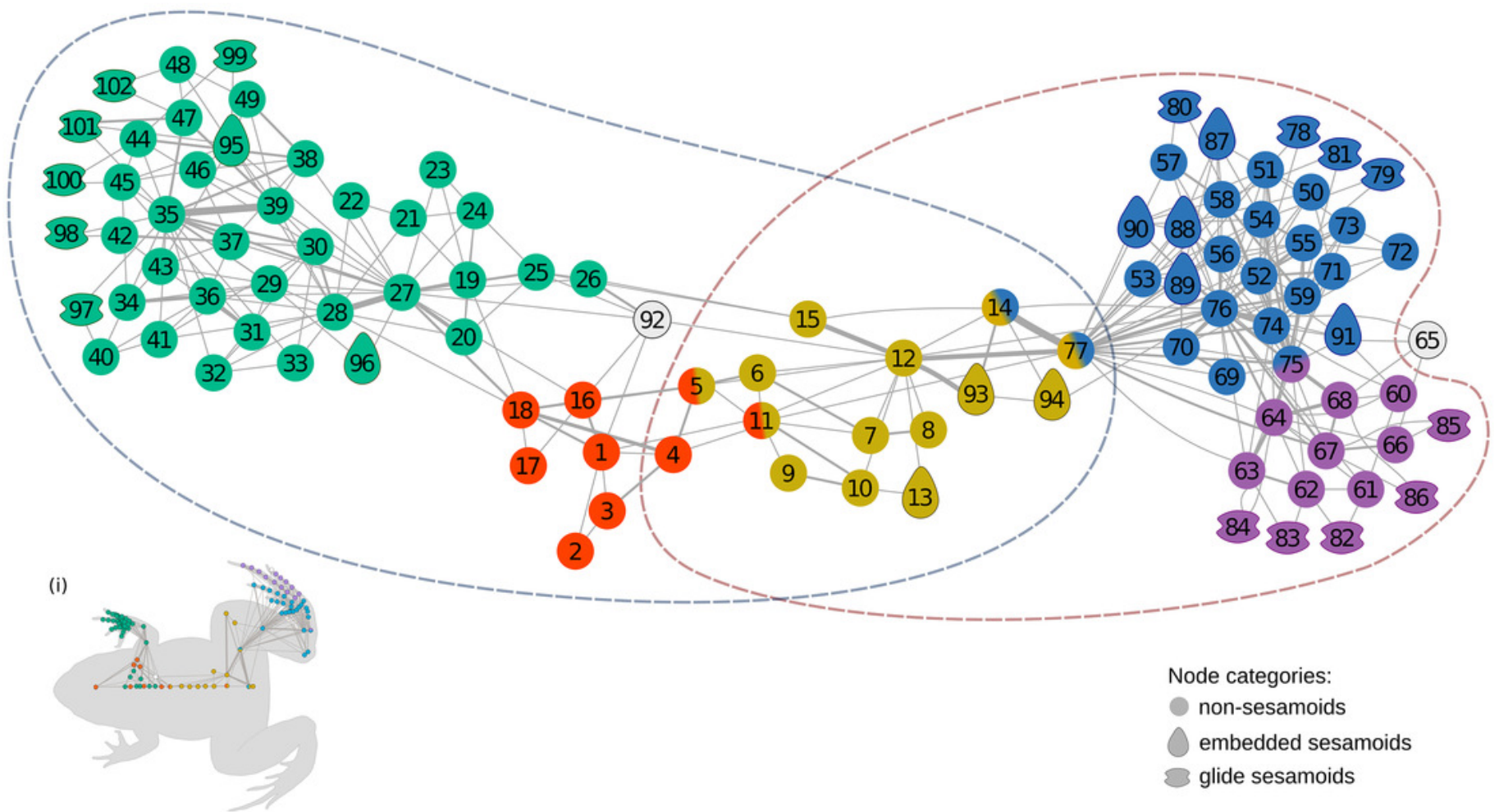




\section{Figure 2}

Leptodactylus latinasus skeleton with the pieces colored according to network modules.

A. Dorsal view. B. ventral view. The fascia dorsalis (pectoral-forelimb module) is represented in a non-saturated grey color to allow the visualization of overlapped structures. Color references: pectoral-forelimb module (green), axial-scapular module (orange), axial-pelvic module (yellow), hindlimb module (blue), IV-V toes module (purple), and homeless pieces (grey). Mix colored pieces are simultaneously members of two modules.
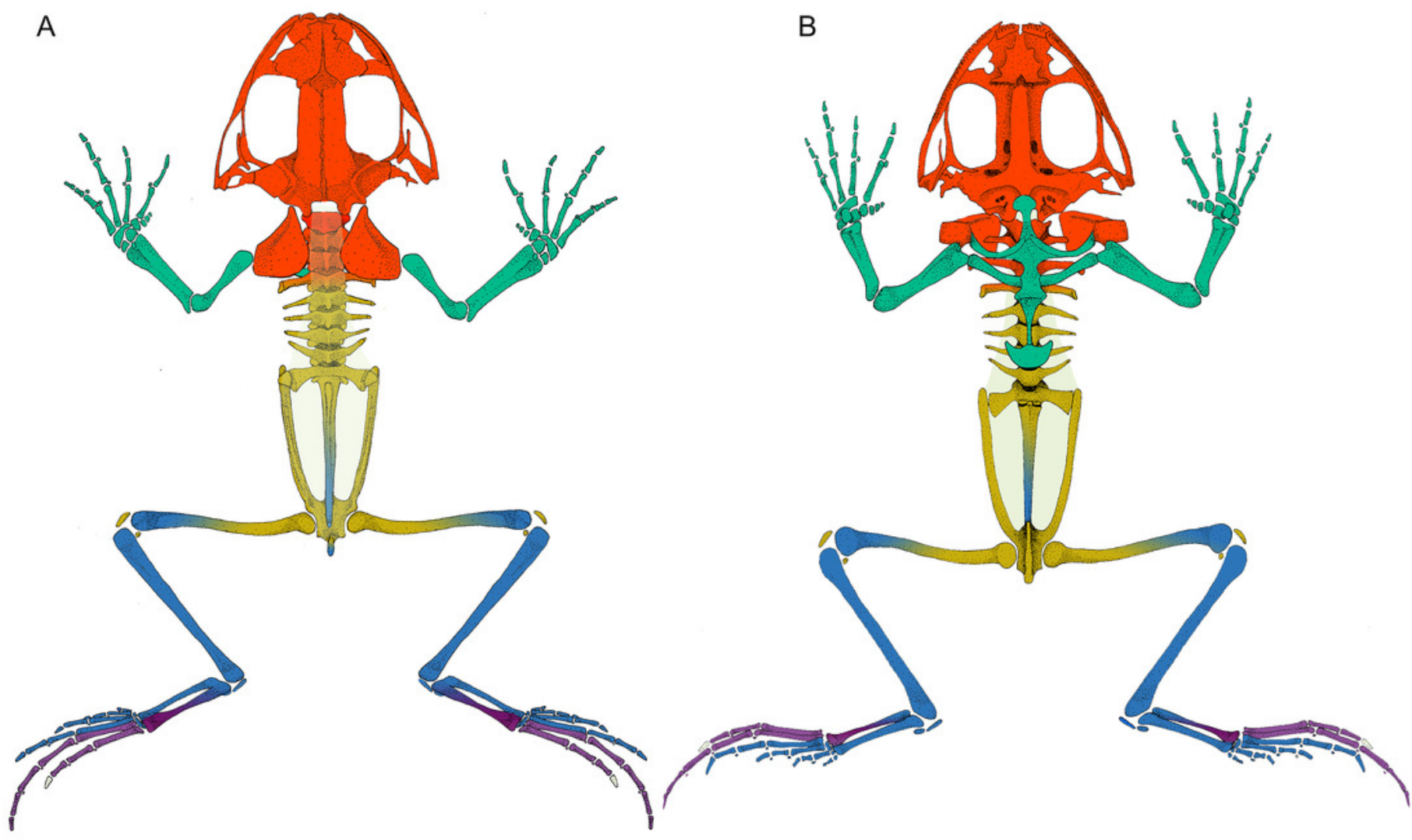


\section{Figure 3}

Schematic representation and node parameter values of each node type in the network.

A. Ventral view of the left hand of Leptodactylus latinasus showing the canonical bones (light purple), the palmar sesamoid (light blue) interphalangeal and metacarpophalangeal glide sesamoids (pink). B. Detail of the palmar sesamoid (embedded type) with the flexor tendons of the digits. C. Detail of an interphalangeal glide associated with a flexor tendon. D. Boxplots of degree values by node category. E. Boxplots of betweenness values by node category. F. Boxplots of closeness values by node category. G. Boxplots of eigen-centrality values by node category. Abbreviations: CSE: Canonical Skeletal Element (light purple); ES: embedded sesamoid (light blue); GS: glide sesamoid (pink). 
A
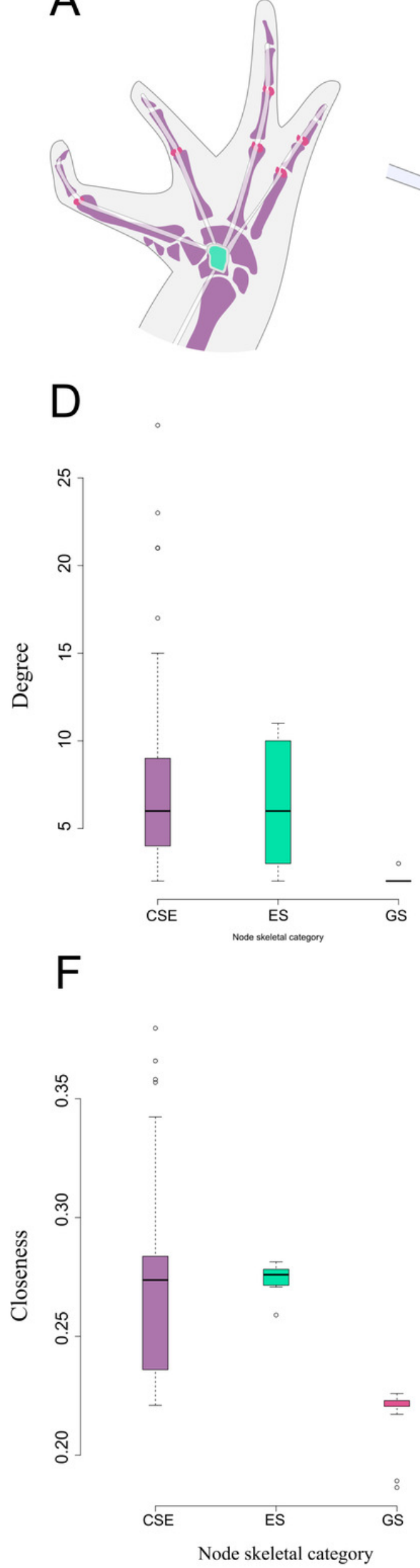

B

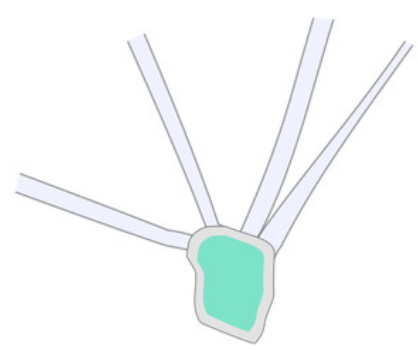

C
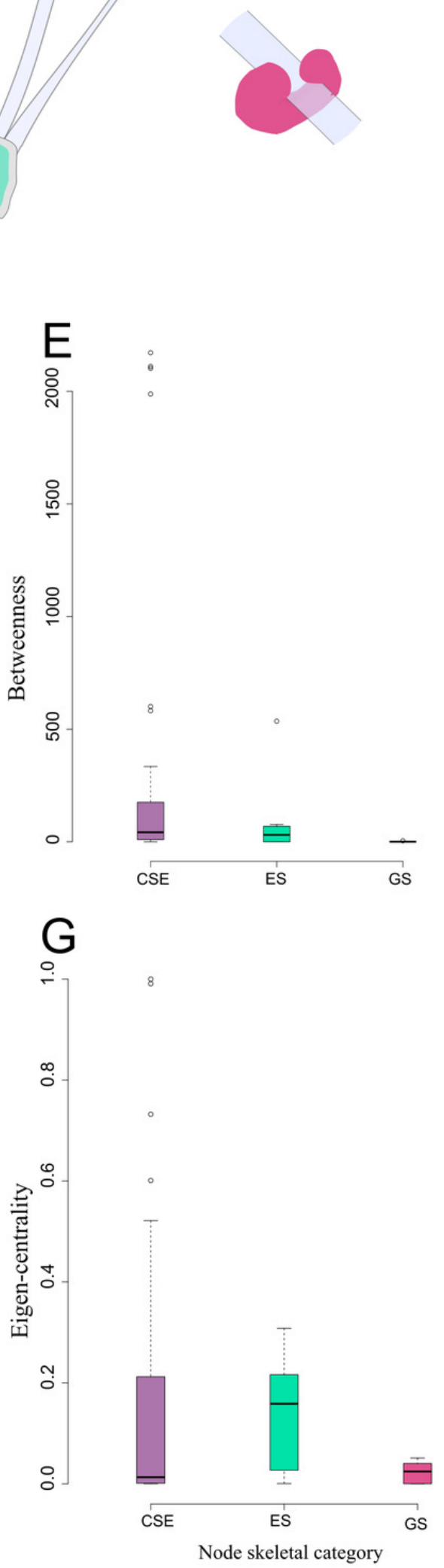